\title{
A novel method for spectrophotometric determination of pregabalin in pure form and in capsules
}

\author{
Alka Bali and Prateek Gaur
}

\begin{abstract}
Background: Pregabalin, a $\gamma$-amino-n-butyric acid derivative, is an antiepileptic drug not yet official in any pharmacopeia and development of analytical procedures for this drug in bulk/formulation forms is a necessity. We herein, report a new, simple, extraction free, cost effective, sensitive and reproducible spectrophotometric method for the determination of the pregabalin.

Results: Pregabalin, as a primary amine was reacted with ninhydrin in phosphate buffer pH 7.4 to form blue violet colored chromogen which could be measured spectrophotometrically at $\lambda_{\text {max }} 402.6 \mathrm{~nm}$. The method was validated with respect to linearity, accuracy, precision and robustness. The method showed linearity in a wide concentration range of 50-1000 $\mathrm{Mg} \mathrm{mL}^{-1}$ with good correlation coefficient (0.992). The limits of assays detection was found to be $6.0 \mu \mathrm{g} \mathrm{mL}$ and quantitation limit was $20.0 \mu \mathrm{g} \mathrm{mL}$. The suggested method was applied to the determination of the drug in capsules. No interference could be observed from the additives in the capsules. The percentage recovery was found to be $100.43 \pm 1.24$.

Conclusion: The developed method was successfully validated and applied to the determination of pregabalin in bulk and pharmaceutical formulations without any interference from common excipients. Hence, this method can be potentially useful for routine laboratory analysis of pregabalin.
\end{abstract}

\section{Background}

Pregabalin (PRG), (S)-3-(aminomethyl)-5-methylhexanoic acid (Figure 1), is an antiepileptic and structurally related to the inhibitory neurotransmitter aminobutyric acid (GABA) It was recently approved for adjunctive treatment of partial seizures in adults $[1,2]$ in United States and Europe and for the treatment of neuropathic pain from post therapeutic neuralgia and diabetic neuropathy. Currently, there is no official analytical procedure for pregabalin in any pharmacopeia. Several reports are there in literature for PRG determination based on chromatographic methods, i.e., gas chromatography-mass spectrophotometry (GC-MS), LC-MS-MS [3,4], HPLC [5-7] coupled with varying detection techniques like tandem mass spectrometry [8], fluorometry [9] and enantiospecific analysis [10]. These methods may involve procedural variations including pre- and post- column

\footnotetext{
* Correspondence: alka.bali@rediffmail.com

University Institute of Pharmaceutical Sciences, Panjab University, Chandigarh,160014, India
}

derivatization [10]. Recently, capillary electrophoresis and nuclear magnetic resonance technique was reported for PRG involving complexation with cyclodextrins [11]. All these are complex trace analysis techniques most of which have been employed for PRG determination in biological fluid samples. However, routine analysis of the drug in bulk powder and pharmaceutical preparations in research laboratories and pharmaceutical industry requires a relatively uncomplicated and a more cost effective method like UV/visible spectrophotometry or spectrofluorometry. Pregabalin, as such, has a poor UV/ visible absorbance profile (Figure 2) and very few reported methods have relied on generation of a chromophoric product by reaction of the drug with some suitable reagent. Considering the limited literature reports available in this area [12-14], we found it very pertinent to investigate and develop a novel spectrophotometric method for determination of pregabalin in bulk powder and pharmaceutical preparations. Ninhydrin has been used as a chromogenic agent in spectrophotometric 


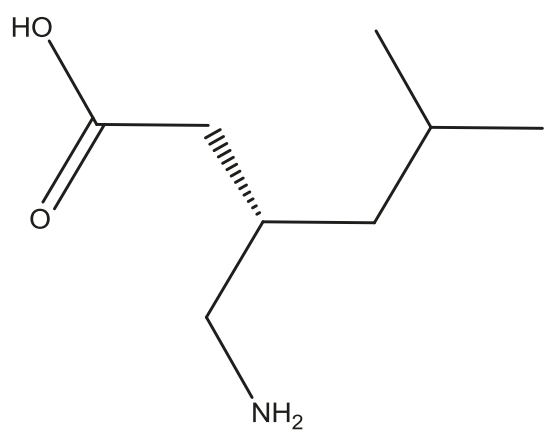

\section{Pregabalin}

\section{(S)-3-(Aminomethyl)-5- methylhexanoic acid}

Figure 1 Chemical structure of pregabalin.

analysis of several amino acids, peptides and amines [15]. The present study describes the evaluation of ninhydrin as a chromogenic reagent in the development of simple and a rapid spectrophotometric method for the determination PGB in its pharmaceutical dosage forms. The procedure does not involve any extraction step with any organic solvent and can be directly carried out in phosphate buffer $\mathrm{pH}$ 7.4 which makes it ideal for routine analysis of the drug in bulk or in pharmaceutical formulations.

\section{Results and discussion Method}

In our efforts to design a novel spectrophotometric method for quantification of pregabalin, we investigated its derivatization with ninhydrin ((triketohydrindene hydrate) for generation of a chromophoric product. Figure 3 shows the $\mathrm{UV} /$ visible spectrum of the chromophoric derivative. The procedure involves formation of purple colored product by reaction of pregabalin with ninhydrin by heating at a temperature of $70-75^{\circ} \mathrm{C}$ for 20 minutes. Reaction of ninhydrin

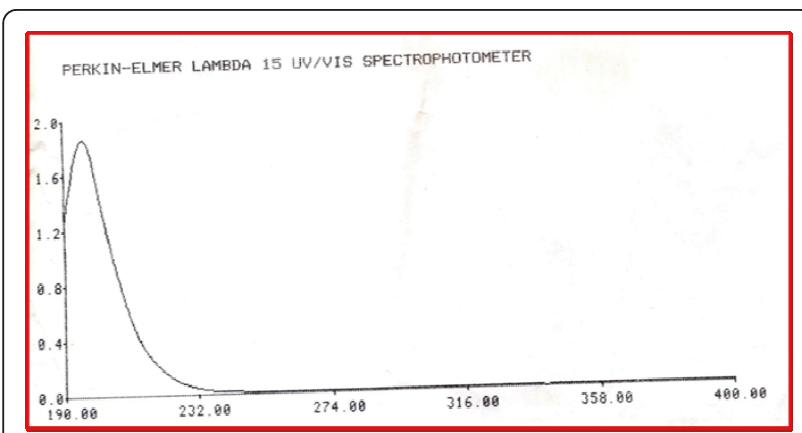

Figure 2 UV/Visible scan of pregabalin without derivatization $\left(\lambda_{\max } 196.2 \mathrm{~nm}\right)$.
1 with amines, alpha amino acids, peptides, and proteins yields an aldehyde with one carbon atom less than the alpha-amino acid; and carbon dioxide in stoichlometric amounts and varying amounts of ammonia, hydrindantin and a chromophoric compound known as Ruhemann's Purple (2-(3-hydroxy-1-oxo- $1 \mathrm{H}$-inden-2-ylimino)- $2 \mathrm{H}$ indene-1,3-dione). This pigment serves as the basis of detection and quantitative estimation of alpha-amino acids. ${ }^{13}$ Mechanism proposed (Figure 4 ) for the reaction involves removal of a water molecule from ninhydrin hydrate $\mathbf{1}$ to generate 1,2,3-indantrione $\mathbf{2}$ in the first step, which then, forms a Schiff's base with the amino group of pregabalin resulting in the ketimine 3 . Removal of the aldehyde $\mathrm{RCHO}$ generates an intermediate amine 4 (2-amino1,3-indandione). Condensation of this intermediate amine with another molecule of ninhydrin follows to form the expected chromophore 5 (Ruhemann's Purple). The ratedetermining step in the entire sequence of the ninhydrin reaction is the nucleophilic-type displacement of a hydroxy group of ninhydrin hydrate by a non-protonated amino group.

\section{Effect of $\mathrm{pH}$}

Of the buffers investigated (acetate buffer, phosphate buffer), colour development was noted in case of phosphate buffer. The optimum buffer $\mathrm{pH}$ was found to be 7.4 and lower $\mathrm{pH}$ ranges resulted in an insufficient colour development.

\section{Effect of reagent concentration}

The addition of $1.0 \mathrm{~mL}$ of ninhydrin solution $(0.2 \% \mathrm{w} / \mathrm{v})$ was sufficient to obtain the maximum and reproducible absorbance values for the various concentration ranges of PGB. Smaller amounts resulted in incomplete reaction. Further increase in the concentration had no significant effect on complex formation, although absorbance increased slightly owing to the reagent background used.

\section{Effects of temperature and heating time}

The effects of temperature and heating time on the formation of the coloured complex were also optimized. At room temperature, the addition of ninhydrin did not lead to the formation of any coloured product and higher temperatures were required to accelerate the reaction. The colour intensity increased with increasing temperature and maximum absorbance was obtained following heating on a water bath at a temperature of $70-75^{\circ} \mathrm{C}$ for 20 minutes. Further heating caused no appreciable change in the colour. The complex obtained was highly stable for more than $6 \mathrm{~h}$.

\section{Validation}

The method was validated with respect to linearity and range, accuracy and precision, limit of detection (LOD) 
PERKIN-ELMER LAMBDA 15 UV/VIS SPECTROPHOTOMETEF

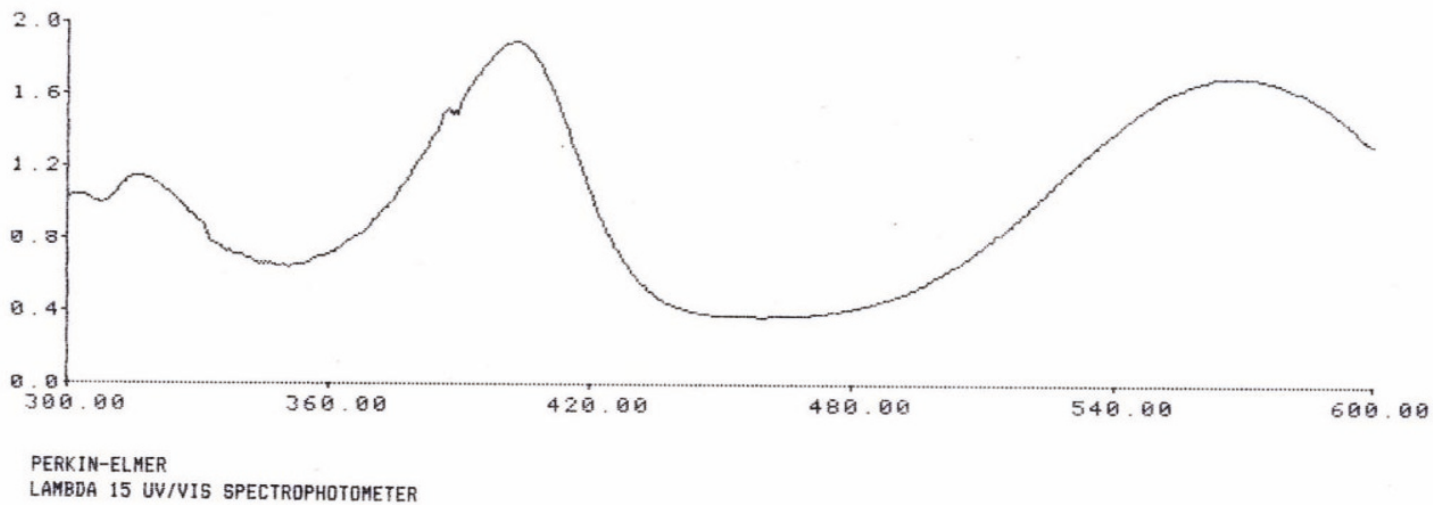

Figure $3 \mathrm{UV} /$ Visible scan of the chromophoric product of pregabalin $\left(\lambda_{\max } 402.6 \mathrm{~nm}\right)$

and limit of quantification (LOQ), selectivity and robustness. The developed method was validated for the pure drug as well as marketed formulation of pregabalin (Pregabalin 75; Torrent pharmaceuticals) and the various validation parameters are shown in Table 1.

\section{Linearity and range}

The regression plots showed compliance with Beer Lambert's law (linearity) in the concentration range of 50 $\mu \mathrm{g} / \mathrm{mL}-1000 \mu \mathrm{g} / \mathrm{mL}$ with a correlation coefficient $\left(\mathrm{r}^{2}\right)$ of 0.992. The standard plot is given in Figure 5. Table 1 summarizes the performance data and statistical parameters for the proposed method including concentration ranges, linear regression equation, correlation coefficient, molar absorptivity, Sandell sensitivity limit and these indicate a good linearity over the working concentration ranges.

\section{Precision}

Precision were investigated by analyzing different concentrations of pregabalin $(0.2-1.4 \mathrm{mg} / \mathrm{mL})$ in three independent replicates on the same day (intra-day precision) and on three consecutive days (inter-day precision). The data is represented as relative standard deviation (RSD)

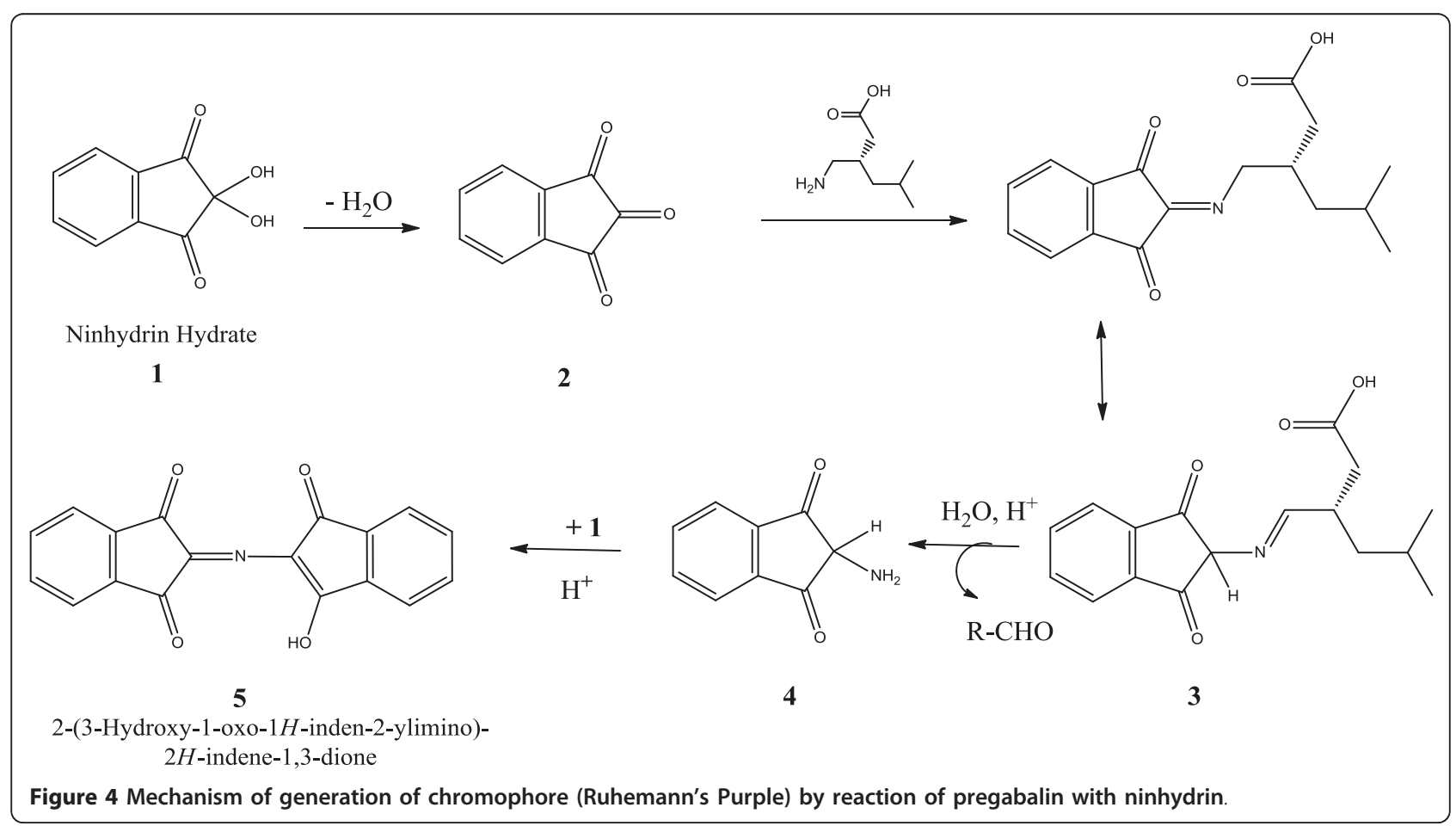


Table 1 Validation data for determination of pregabalin by proposed method

\begin{tabular}{cc}
\hline Validation parameter & Results \\
\hline Absorption maximum & $402.6 \mathrm{~nm}$ \\
Regression equation & $\mathrm{y}=1.908 \times-0.056$ \\
Slope & 1.908 \\
Intercept & 0.056 \\
Beer's law limit $\left(\mu \mathrm{gL}^{-1}\right)$ & $50-1000$ \\
Molar Absorptivity $\left(\mathrm{L} / \mathrm{mol}^{-1} / \mathrm{cm}^{-1}\right)$ & 1198.4 \\
Sandell's sensitivity $\left(\mu \mathrm{gg} / \mathrm{cm}^{2} / 0.001 \mathrm{~A} . \mathrm{U}\right)$ & 0.1328 \\
Coefficient of correlation(r) & 0.992 \\
Limit of detection $(\mathrm{LOD})$ & $0.006 \mathrm{mg} / \mathrm{mL}$ \\
Limit of quantification (LOQ) & $0.02 \mathrm{mg} / \mathrm{mL}$ \\
Accuracy & $100.43 \pm 1.24$ \\
Precision & Intra-day $=0.62$ \\
& Inter-day $=0.61$ \\
Robustness & Less than $1 \%$ \\
\% Purity & $99.8 \%$ \\
\hline
\end{tabular}

and results have been shown in Table 2. Low relative standard deviation (RSD) values for intra- day and interday analysis indicate good precision of the method.

\section{Accuracy}

The accuracy of the method signifies the closeness of the measured value to the true value for the sample. To determine the accuracy of the proposed method, different levels of drug concentrations were prepared from independent stock solutions and analyzed. To provide an additional support to the accuracy of the developed assay method, a standard addition method was employed, which involved the addition of different concentrations of pure drug to a known pre analyzed dilution of the pure drug as well as formulation sample and the total concentration was determined using the proposed method. Accuracy was assessed as the percentage relative error $E_{r}$ and mean \% recovery. The percentage recovery of the added pure drug was calculated as:

$$
\% \text { recovery }=\left[\left(\mathrm{C}_{\mathrm{t}}-\mathrm{C}_{\mathrm{i}}\right) / \mathrm{C}_{\mathrm{a}}\right] \times 100,
$$

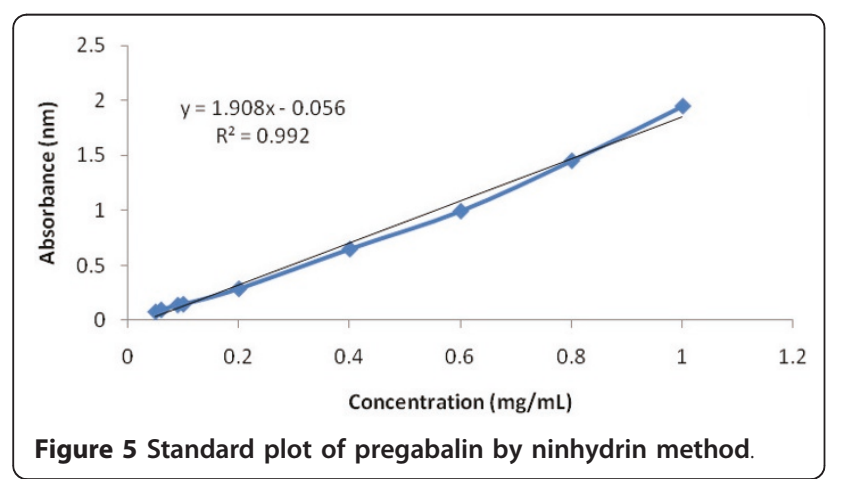

Table 2 Precision of the proposed methods for the analysis of pregabalin

\begin{tabular}{lcccc}
\hline Pregabalin (mg/ml) & \multicolumn{2}{c}{ Within day, $\mathbf{n}=\mathbf{3}$} & \multicolumn{2}{c}{ Between day, $\mathbf{n}=\mathbf{3}$} \\
\cline { 2 - 5 } & Mean \pm SD & RSD (\%) & Mean \pm SD & RSD (\%) \\
\hline 0.2 & $0.288 \pm 0.14$ & 0.49 & $0.287 \pm 0.0005$ & 0.0020 \\
0.4 & $0.551 \pm 0.28$ & 0.51 & $0.551 \pm 0.0004$ & 0.0007 \\
0.6 & $0.890 \pm 0.42$ & 0.48 & $0.890 \pm 0.0003$ & 0.0004 \\
0.8 & $1.451 \pm 0.56$ & 0.39 & $1.452 \pm 0.0005$ & 0.0004 \\
1.0 & $1.989 \pm 0.71$ & 0.36 & $1.990 \pm 0.0003$ & 0.0002 \\
\hline
\end{tabular}

Where

$\mathrm{C}_{\mathrm{t}}$ is the total drug concentration measured after standard addition;

$\mathrm{C}_{\mathrm{i}}$ drug concentration in the formulation sample;

$\mathrm{C}_{\mathrm{a}}$, drug concentration added.

The percentage relative error was calculated as: $\mathrm{E}_{\mathrm{r}} \%=$ [(found - added $) /$ added $] \times 100$

Recovery values from standard addition method followed for the bulk drug analysis ranged from 96.5 to $100.3 \%$ (Table 3). Recovery studies with marketed formulation returned values ranging from 97.09 to $99.74 \%$ (Table 4).

\section{Interference}

Satisfactory values of the mean recovery values $\pm \mathrm{SD}$, RSD \% and $E_{r} \%$ in recovery studies in drug formulation (Table 4) revealed that there is no potential interference of the excipients listed by the manufacturer, i.e., talc, lactose monohydrate and maize starch. This may be attributed to the dependence of the reaction in the proposed method on the presence of a primary aliphatic amino group in the drug molecule which is not present in any of these excepients.

\section{Limit of detection (LOD) and limit of quantitation (LOQ)}

LOD and LOQ of the method were established using calibration standards (Table 1). LOD and LOQ were calculated as $3.3 \sigma / \mathrm{s}$ and $10 \sigma / \mathrm{s}$, respectively, as per $\mathrm{ICH}$ definitions, where, $\sigma$ is the mean standard deviation of replicate determination values under the same conditions as the sample analysis in the absence of the analyte (blank determination), and $\mathrm{s}$ is the sensitivity, namely the slope of the calibration graphs.

\section{Robustness}

Repeatability is based on the results of the method operating over short interval of time under same conditions. Robustness was examined by evaluating the small variations in different experimental conditions such as heating temperatures $\left( \pm 2^{\circ} \mathrm{C}\right)$, working wavelengths, volume and concentration of reagents. Three replicate determinations at six different concentration levels of the drugs were carried out. The within-day RSD values were 
Table 3 Results of recovery studies with pure drug pregabalin by standard addition method

\begin{tabular}{cccccc}
\hline Initial Conc. $(\mathbf{m g} / \mathbf{m L})$ & Volume $(\mathbf{m L})$ & Amount added $(\boldsymbol{\mu g})$ & Amount recovered $(\boldsymbol{\mu g})$ & ${\text { Recovery }(\%) \pm \mathrm{SD}^{\mathbf{a}}}_{\text {RSD }^{(\%)}}$ \\
\hline 0.2 & 5.0 & 100.0 & 98.7 & $98.70 \pm 0.890$ & 0.91 \\
0.4 & 5.0 & 100.0 & 96.5 & $96.50 \pm 0.923$ & 0.95 \\
0.6 & 5.0 & 100.0 & 99.7 & $99.70 \pm 0.624$ & 0.63 \\
0.8 & 5.0 & 100.0 & 100.3 & $100.3 \pm 1.600$ & 1.57 \\
1.0 & 5.0 & 100.0 & 98.1 & $98.10 \pm 1.718$ \\
\hline
\end{tabular}

${ }^{a}$ Average of determinations carried out in triplicate.

found to be less than $0.6 \%$ indicating that the proposed method has reasonable robustness.

\section{Stability}

The stability of the final sample solutions was examined by their absorbance values and responses were found to be stable for at least 6 hours at room temperature.

\section{Analysis of marketed formulation (Pregabalin capsules)}

Table 5 gives the results of the assay for pregabalin carried out on marketed formulation by the proposed method and revealed that there is close agreement between the results obtained by the proposed methods and the label claim. The recovered drug content was found to be $99.48 \%$.

\section{Analytical applications}

The results with the proposed method for the determination of pregabalin in its pharmaceutical formulation
(Pregabalin75 capsules) suggest satisfactory recovery. Further, standard addition technique followed to check the validity of the method have given good recoveries of the drug in presence of formulation suggesting a noninterference from formulation excepients. Hence, this method can be recommended for adoption in routine analysis of pregabalin in in quality control laboratories.

\section{Conclusion}

The method proposed is simple, rapid, inexpensive and sensitive for the determination of pregabalin in bulk as well as in marketed form (capsules). There is no requirement of any sophisticated apparatus as in chromatographic methods. Omission of an extraction step with organic solvents is an added advantage. The method has been validated in terms of its sensitivity, simplicity, reproducibility, precision, accuracy and stability of the coloured species for $\geq 6$ h suggesting its suitablility for the routine analysis of PGB in pure form (in bulk

Table 4 Recovery studies with pregabalin capsules by standard addition method

\begin{tabular}{|c|c|c|c|c|c|c|}
\hline Initial Conc. (mg/mL) & Volume $(\mathrm{mL})$ & Amount of PGB added $(\mu \mathrm{g})$ & Amount recovered $(\mu \mathrm{g})$ & Recovery (\%) $\pm \mathrm{SD}^{\mathrm{a}}$ & $\mathrm{E}_{\mathrm{r}} \%$ & RSD (\%) \\
\hline 0.05 & 5.0 & 50.0 & 49.5 & $98.85 \pm 0.145$ & -1.14 & 0.14 \\
\hline 0.05 & 5.0 & 100.0 & 99.5 & $99.09 \pm 0.370$ & -0.91 & 0.37 \\
\hline 0.05 & 5.0 & 200.0 & 199.1 & $99.45 \pm 0.124$ & -0.550 & 0.13 \\
\hline 0.1 & 5.0 & 50.0 & 49.1 & $98.71 \pm 0.471$ & -1.29 & 0.47 \\
\hline 0.1 & 5.0 & 100.0 & 98.1 & $98.16 \pm 0.271$ & -1.83 & 0.28 \\
\hline 0.1 & 5.0 & 200.0 & 98.1 & $98.16 \pm 0.271$ & -1.83 & 0.28 \\
\hline 0.2 & 5.0 & 50.0 & 49.0 & $98.44 \pm 0.426$ & -1.55 & 0.43 \\
\hline 0.2 & 5.0 & 100.0 & 99.7 & $99.67 \pm 0.186$ & -0.32 & 0.19 \\
\hline 0.2 & 5.0 & 200.0 & 199.0 & $99.49 \pm 0.165$ & -0.510 & 0.17 \\
\hline 0.4 & 5.0 & 50.0 & 49.7 & $99.27 \pm 0.251$ & -0.73 & 0.25 \\
\hline 0.4 & 5.0 & 100.0 & 97.6 & $97.54 \pm 0.196$ & -2.45 & 0.20 \\
\hline 0.4 & 5.0 & 200.0 & 199.7 & $99.74 \pm 0.123$ & -0.253 & 0.12 \\
\hline 0.6 & 5.0 & 50.0 & 49.8 & $99.07 \pm 0.483$ & -0.92 & 0.48 \\
\hline 0.6 & 5.0 & 100.0 & 98.2 & $98.29 \pm 0.134$ & -1.70 & 0.14 \\
\hline 0.6 & 5.0 & 200.0 & 198.9 & $98.71 \pm 0.646$ & -1.287 & 0.65 \\
\hline 0.8 & 5.0 & 50.0 & 49.1 & $98.62 \pm 0.479$ & -1.38 & 0.48 \\
\hline 0.8 & 5.0 & 100.0 & 99.2 & $99.25 \pm 0.098$ & -0.74 & 0.10 \\
\hline 0.8 & 5.0 & 200.0 & 198.2 & $99.22 \pm 0.136$ & -0.777 & 0.14 \\
\hline 1.0 & 5.0 & 50.0 & 50.0 & $99.49 \pm 0.495$ & -0.51 & 0.49 \\
\hline 1.0 & 5.0 & 100.0 & 97.0 & $97.09 \pm 0.147$ & -2.91 & 0.15 \\
\hline 1.0 & 5.0 & 200.0 & 199.5 & $99.66 \pm 0.198$ & -0.333 & 0.20 \\
\hline
\end{tabular}

${ }^{a}$ Average of determinations carried out in triplicate. 
Table 5 Assay results for pregabalin determination in capsule formulation

\begin{tabular}{|c|c|c|c|}
\hline Formulation & Label claim (mg) & Mean Recovery $(\mathrm{mg}) \pm S D^{a}$ & Mean $\%$ Recovery $^{\mathrm{a}} \pm \mathrm{SD}^{\mathrm{a}}$ \\
\hline Pregabalin 75 (Torrent Pharmaceuticals) & 75 & $74.61 \pm 0.55$ & $99.48 \pm 0.73$ \\
\hline
\end{tabular}

${ }^{a}$ Average of nine determinations, three each with starting powder amount equivalent to 30, 60 and $90 \mathrm{mg}$ pregabalin.

analysis) as well as pharmaceutical formulations without interference from excipients.

\section{Experimental Apparatus}

All absorption spectra were recorded using a Perkin Elmer lambda 15 UV-Visible spectrophotometer (German) with a scanning speed of $60 \mathrm{~nm} / \mathrm{min}$ and a band width of $2.0 \mathrm{~nm}$, equipped with $10 \mathrm{~mm}$ matched quartz cells. A CyberScan pH 510 (Eutech instruments) pH meter was used for checking the $\mathrm{pH}$ of buffer solutions.

\section{Materials and reagents}

All chemicals and materials were of analytical grade and were purchased from Qualigens fine chemicals, Mumbai, India. All solutions were freshly prepared in double distilled water.

\section{Pure samples}

Pregabalin (PGB) pure grade was graciously provided as a gift samples by Vardhman Chemtech limited, Derabassi, Punjab, India.

\section{Market samples}

Pregabalin75 capsules (label amount 75 mg PGB/Capsule) Torrent Pharmaceuticals were purchased from the market.

\section{Preparation of phosphate buffer $\mathrm{pH} 7.4$}

Phosphate buffer $\mathrm{pH} 7.4$ was prepared by mixing 250 $\mathrm{mL}$ of $0.2 \mathrm{M}$ potassium dihydrogen phosphate with $195.5 \mathrm{ml}$ of $0.2 \mathrm{M} \mathrm{NaOH}$ and making up the volume to $1000 \mathrm{ml}$ with distilled water. The $\mathrm{pH}$ of the buffer was adjusted to 7.4 using a precalibrated $\mathrm{pH}$ meter.

\section{Standard Stock solutions}

Stock solution of pregabalin $(2 \mathrm{mg} / \mathrm{ml})$ was prepared by dissolving $200 \mathrm{mg}$ of pregabalin in $100 \mathrm{~mL}$ of phosphate buffer ( $\mathrm{pH} 7.4)$.

\section{Preparation of ninhydrin solution}

The $0.2 \%$ solution of ninhydrin was prepared by dissolving $200 \mathrm{mg}$ of ninhydrin in $100 \mathrm{ml}$ of ethanol and was kept in an amber colored bottle.

\section{Method}

Standard plot

Different aliquots were taken from the stock solution $(2 \mathrm{mg} / \mathrm{ml})$ and diluted with phosphate buffer $\mathrm{pH} 7.4$ to prepare a series of concentrations ranging from 50 to $1000 \mu \mathrm{g} / \mathrm{mL}$ of pregabalin. To $5.0 \mathrm{~mL}$ of these aliquots taken in stoppered tubes, $1.0 \mathrm{~mL}$ of ninhydrin solution $(0.2 \% \mathrm{w} / \mathrm{v})$ was added and heated on a water bath at a temperature of $70-75^{\circ} \mathrm{C}$ for 20 minutes. The tubes were kept covered to avoid the loss of solvent due to evaporation. After cooling the solution to room temperature, the absorbance values were measured in triplicate at $402.6 \mathrm{~nm}$ against mixture of $5.0 \mathrm{~mL}$ phosphate buffer (pH 7.4) and $1.0 \mathrm{~mL} \mathrm{0.2 \%} \mathrm{ninhydrin} \mathrm{as} \mathrm{reagent} \mathrm{blank.}$ The calibration graph was obtained by plotting the absorbance values at the $\lambda_{\max }$ of the drug $(402.6 \mathrm{~nm})$ against corresponding concentration values and compliance with Beer Lambert's law was assessed.

\section{Analysis of pharmaceutical formulation Preparation of capsule sample solution}

The contents of twenty capsules were mixed and weighed accurately. Separate quantities of the powder equivalent to $30 \mathrm{mg}, 60 \mathrm{mg}$ and $90 \mathrm{mg}$ of PRG were transferred into a $100 \mathrm{~mL}$ volumetric flasks, dissolved in water, and sonicated for $5 \mathrm{~min}$., the volume was then completed with water, shaken well for $5 \mathrm{~min}$. and filtered into a dry flask. To $5.0 \mathrm{~mL}$ aliquots of the filtrate taken in stoppered tubes, $1.0 \mathrm{~mL}$ of ninhydrin solution $(2.0 \% \mathrm{w} / \mathrm{v})$ was added and solution heated on a water bath at a temperature of $70-75^{\circ} \mathrm{C}$ for 20 minutes. Solutions were cooled to room temperature and the absorbance values noted in triplicate at $402.6 \mathrm{~nm}$ against reagent blank.

\section{Authors' contributions}

$A B$ conceived and supervised this research work, participated in the data analysis and drafted the manuscript. PG carried out the drug analysis and the validation studies.

\section{Competing interests}

The authors declare that they have no competing interests.

Received: 19 July 2011 Accepted: 7 October 2011

Published: 7 October 2011

\section{References}

1. Tassone DM, Boyce E, Guyer J, Nuzum D: Pregabalin: a novel gammaaminobutyric acid analogue in the treatment of neuropathic pain, partial-onset seizures, and anxiety disorders. Clin Ther 2007, 29(1):26-48.

2. Hamandi K, Sander JW: Pregabalin: a new antiepileptic drug for refractory epilepsy. Seizure 2006, 15(2):73-78.

3. Vaidya W, Yetal SM, Roy SMN, Gomes NA, Joshi SS: LC-MS-MS Determination of Pregabalin in Human Plasma. Chromatographia 2007, 66(11-12):925-928.

4. Mandal U, Sarkar AK, Gowda KV, Agarwal S, Bose A, Bhaumik U, Ghosh D, Pal TK: Determination of Pregabalin in Human Plasma Using LC-MS-MS. Chromatographia 2008, 67(3-4):237-243. 
5. David B, Millington C: Analysis of Pregabalin at Therapeutic Concentrations in Human Plasma/Serum by Reversed-Phase HPLC. Ther Drug Monit 2005, 27(4):451-456.

6. Gujral RS, Manirul Haque SK, Kumar S: A novel method for the determination of pregabalin in bulk pharmaceutical formulations and human urine samples. Afr J Pharm Pharmacol 2009, 3(6):327-334.

7. Kasawar GB, Farooqui MN: Development and validation of HPLC method for the determination of pregabalin in capsules. Indian J Pharm Sci 2010, 72(4):517-519.

8. Shah GR, Ghosh C, Thaker BT: Determination of pregabalin in human plasma by electrospray ionisation tandem mass spectroscopy. J Adv Pharm Tech Res 2010, 1:354-357.

9. Vermeij TAC, Edelbroek PM: Simultaneous high-performance liquid chromatographic analysis of pregabalin, gabapentin and vigabatrin in human serum by precolumn derivatization with o-phtaldialdehyde and fluorescence detection. J Chromatogr B Analyt Technol Biomed Life Sci 2004, 810(2):297-303

10. Jadhav AS, Pathare DB, Shingare MS: Validated enantioselective LC method, with precolumn derivatization with Marfey's reagent, for analysis of the antiepileptic drug pregabalin in bulk drug samples. Chromatographia 2007, 65(3-4):253-256.

11. Béni S, Sohajda T, Neumajer G, Iványi R, Szente L, Noszál B: Separation and characterization of modified pregabalins in terms of cyclodextrin complexation, using capillary electrophoresis and nuclear magnetic resonance. J Pharm Biomed Anal 2010, 51(4):842-852.

12. Gujral RS, Manirul Haque SK, Sharma P: Development and validation of pregabalin in bulk, pharmaceutical formulations and in human urine samples by UV spectrophotometry. Int J Biomed Sci 2009, 5(2):175-180

13. Onal A, Olcay S: Spectrophotometric and spectrofluorimetric methods for the determination of pregabalin in bulk and pharmaceutical preparation. Spectrochim Acta A 2009, 72(1):68-71.

14. Rasha Abdel-Aziz Shaalan: Spectrofluorimetric and Spectrophotometric Determination of Pregabalin in Capsules and Urine Samples. Int I Biomed Sci 2010, 6(3):260-267.

15. Friedman M: Applications of the Ninhydrin Reaction for Analysis of Amino Acids, Peptides, and Proteins to Agricultural and Biomedical Sciences. J Agric Food Chem 2004, 52(3):385-406.

doi:10.1186/1752-153X-5-59

Cite this article as: Bali and Gaur: A novel method for spectrophotometric determination of pregabalin in pure form and in capsules. Chemistry Central Journal 2011 5:59.

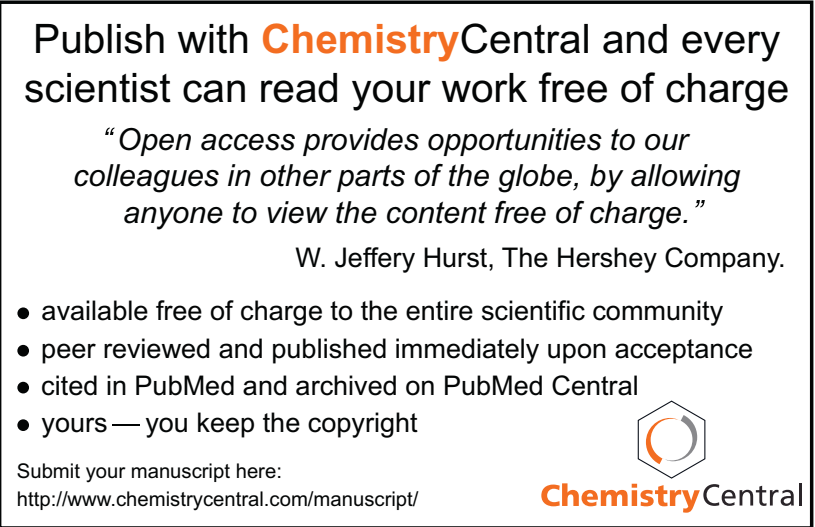

